\title{
Development of Eye Phantoms for Mimicking the Structural Deformation of the Human Cornea Accompanied With Intraocular Pressure Alteration
}

Han Saem Cho

Korea Research Institute of Standards and Science

Sae Chae Jeoung ( $\nabla$ scjeoung@kriss.re.kr)

Korea Research Institute of Standards and Science

\section{Research Article}

Keywords: eye phantoms, intraocular pressure (IOP), human cornea, structural deformation, central corneal thickness (CCT), corneal radius of curvature (CRC)

Posted Date: February 24th, 2021

DOI: https://doi.org/10.21203/rs.3.rs-224906/v1

License: (c) (i) This work is licensed under a Creative Commons Attribution 4.0 International License.

Read Full License 


\title{
Development of eye phantoms for mimicking the structural deformation of the human cornea accompanied with intraocular pressure alteration
}

\author{
Han Saem Cho and Sae Chae Jeoung* \\ Korea Research of Standards and Science, Daejeon, 34113, Korea \\ Correspondence e-mail: scjeoung@kriss.re.kr
}

\begin{abstract}
We developed a fully continuous eye phantom to better understand structural deformation of the cornea under varying intraocular pressure (IOP). The IOP-induced deformation and tension of the eye phantom were investigated using optical coherence tomography and non-contact tonometer readings, respectively. A fixed-cornea eye phantom, which featured a soft cornea, was also used for comparison. We evaluated the corneal structural changes between the two different types of eye phantoms by estimating the central corneal thickness (CCT) and corneal radius of curvature (CRC). For the eye phantom with an initial CCT of $0.55 \mathrm{~mm}$, which is close to the average human CCT, CRC of the fully continuous eye phantoms showed a positive correlation to true IOP, while the CRC of a fixed-cornea eye phantom had a negative correlation. Non-contact tonometry readings for fixed-cornea eye phantoms were higher than those of full-eye phantoms due to the structural and mechanical characteristics. Considering the results from in vitro studies on enucleated human eyeballs, a fully continuous eye phantom is a more suitable choice for mimicking human IOP than a fixed-cornea eye phantom. Use of a more reliable eye phantom for accurate estimation of IOP using tonometry may eventually improve the accuracy of glaucoma screening.
\end{abstract}

\section{INTRODUCTION}

Eye phantoms, which are designed to reproduce characteristics of the human eye, have been developed for various purposes: prosthetics, surgery simulation ${ }^{1-3}$, and testing and evaluation of diagnostic instruments ${ }^{4-}$ 7 , including the optical coherence tomography (OCT) machine and the tonometer. The shapes of eye phantoms vary with their purposes. Intraocular pressure (IOP), which is the fluidic pressure inside the eyeball, is usually measured by tonometry, and this test produces the only measurable value to diagnose glaucoma and monitor disease progression and treatment response. Almost all tonometers estimate IOP by measuring the force required to deform the cornea, because it is not plausible to measure IOP invasively. Hence, it is invaluable to invent an eye phantom to mimic the structural deformation as well as the tension accompanying a change in IOP.

Eye phantoms ${ }^{6-9}$ have been developed specifically for mimicking the human cornea. Anthony et al. ${ }^{6}$ developed an eye phantom used for testing of a glaucoma diagnosis device, that was similar to a contact lens. The device made of silicone, but Young's modulus of the cornea, a measure of elasticity, was 
approximately $2.5 \mathrm{MPa}$, which was quite different from that of human subjects. Also inconsistent were the central corneal thickness (CCT) and the corneal radius of curvature (CRC), which were about $0.21 \mathrm{~mm}$ and $8.2 \mathrm{~mm}$, respectively. Hsu et al. ${ }^{7}$ developed an eye phantom for long-term IOP monitoring to test a lenstyped sensor system. This eye phantom mimicked the pressure changes using a micro-pump. Khan et al. ${ }^{8}$ developed an eye phantom with viscoelastic material for cornea elastography measurements, and its CRC was $8.4 \mathrm{~mm}$. Match et al. ${ }^{9}$ developed a silicone eye phantom to characterize the corneal tangent modulus. The CCT and the CRC of this eye phantom were $1.1 \mathrm{~mm}$ and $8 \mathrm{~mm}$, respectively. Because the CCT and the CRC of normal human cornea are in range of $400 \mu \mathrm{m} \sim 600 \mu \mathrm{m}^{10-12}$ and $7 \mathrm{~mm} \sim 8 \mathrm{~mm}^{10,12}$, respectively, we can say that all of the aforementioned phantoms are not quite optimal. Also, these phantoms are modular with a replaceable and flexible cornea. The cornea of these models is made of an elastic polymer to mimic the flexible tissues of a human cornea, while other parts are fabricated with solid materials. The artificial cornea is usually fixed to the rigid parts. These fixed-cornea eye phantoms have the advantage of being easy to fabricate and replace with materials of different structural and mechanical properties. However, there is no previous investigation on a fixed-cornea eye phantom that can mimic accurately the structural deformation of the human eye, which is a single body made of soft and flexible materials. If IOP increases or an external force is applied to the human eyeball, deformation occurs all over the eyeball in order to maintain equilibrium. In contrast, in the case of a fixed-cornea eye phantom, any deformation occurs only in the cornea because the other parts of the eye phantoms are rigid. For this reason, deformation of the fixed-cornea phantom, by IOP changes and/or an external force, would be different from that of a human eye; moreover, as the tension of the cornea is measured from the outside, eventually the estimation of IOP will be affected.

To fulfill the requirements of the international standard for performance testing on a tonometer (ISO 8612), at least 150 human subjects should be tested and there should not be $>5 \%$ of the paired differences between the reference tonometer readings and the test tonometer readings on human subjects are greater than $\pm 5 \mathrm{mmHg}$. It is time-consuming and rather expensive to confirm by performance testing on a tonometer that it meets this standard by conducting relatively large-scale population studies. To solve this issue, it is important to develop eye phantoms in which structural deformation as well as tension measured from the outside, upon applying the IOP, mimics the human eye fairly well. Because most applanation tonometers yield an IOP reading by converting the force needed to flatten the corneal surface of a predetermined area, this force is a function of the stiffness. Thus, it is difficult to estimate the true IOP from the tonometer readings without detailed understanding of the contribution of IOP-induced deformation in addition to that from corneal thickness. Most eye phantoms, which are used for evaluating the performance of tonometers, are usually fixed-cornea eye phantoms. This type of eye phantom mimics deformation in the human eyeball only to a certain extent.

In this study, we report a novel method to fabricate fully continuous eye phantoms made of soft and flexible materials. We also fabricated fixed-cornea eye phantoms and used them for comparative studies. We assessed the validity of these two types of eye phantoms, to mimic human IOP, by measuring their structural deformation and non-contact tonometer (NCT) readings with varying IOP.

\section{RESULTS}

OCT images, as a function of IOP, are shown in Fig. 1(a) and (b) for the fixed-cornea and fully continuous eye phantoms, respectively. The phantoms were designed to have a CCT under zero IOP $\left(\mathrm{CCT}_{0}\right)$ of approximately $0.35 \mathrm{~mm}$. For both eye phantoms, CCT apparently decreased with increasing IOP. The CRC for the fixed-cornea eye phantoms apparently decreased with increasing IOP, whereas an increase in the IOP of the fully continuous eye phantoms resulted in a less prominent decrement in the CRC. To obtain more detailed information on the structural deformation of the eyeball phantoms, we measured OCT images of the cornea with IOP changes $(0 \mathrm{mmHg}-45 \mathrm{mmHg})$ at every $1 \mathrm{mmHg}$ interval, and estimated 
both CCT and CRC. The results for the fixed-cornea and the continuous eye phantoms are shown in Fig. 1(c) and (d), respectively. The CCT decreased linearly as the internal pressure increased. The slope of the plot is almost independent of the type of eye phantom (see Table 1). For fixed-cornea eye phantoms, the average slope was about $-1.72 \pm 0.14 \mu \mathrm{m} / \mathrm{mmHg}$ while for continuous eye phantoms, the slope was about $-1.86 \pm 0.09 \mu \mathrm{m} / \mathrm{mmHg}$. Because the slopes for the two types of eye phantoms are within the standard deviation, it is reasonable to tentatively conclude that the dependence of the CCT on the IOP may be virtually the same between the two models. In addition, $\mathrm{CCT}_{0}$, determined by linear regression, was almost consistent with the initial designs for the CCT.

Meanwhile, as shown in Fig. 1(e) and (f), the dependence of the CRC of the fixed-cornea eye phantoms, accompanied by IOP increment, was quite different from that of the fully continuous eye phantoms. For the fixed-cornea eye phantoms, with the $\mathrm{CCT}_{0}$ of $0.37 \mathrm{~mm}, 0.46 \mathrm{~mm}$, and $0.57 \mathrm{~mm}, \mathrm{CRC}$ decreased as the IOP increased. The degree of CRC decrement apparently reduced with increasing $\mathrm{CCT}_{0}$. The CRC of the fully continuous eye phantom with the $\mathrm{CCT}_{0}$ of $0.37 \mathrm{~mm}$ and $0.46 \mathrm{~mm}$ exhibited a negative correlation with the IOP. However, in the case of fully continuous eye phantoms, with a $\mathrm{CCT}_{0}$ of $0.57 \mathrm{~mm}$, the CRC increased with increasing IOP. It should also be noted that the dependence of CRC on the internal pressure for the fully continuous eye phantom was much less than that for the fixed-cornea phantom. The overall changes in the CRC accompanying an increase in IOP, from $0 \mathrm{mmHg}$ to $45 \mathrm{mmHg}$, for both model eyes are shown in Table 1.

We further investigated the dependence of the tonometer readings on IOP by using an air-puff tonometer. Fig. 2(a) and (b) show the photographs taken during the measurement of NCT readings. As shown in Fig. 2(c), the NCT readings for the fixed-cornea eye phantoms are positively correlated with IOP. Any explanation for the dependence of the NCT readings on the IOP was unavailable while considering the effect of structural deformation on the tonometer readings. We tentatively supposed that the NCT readings might be linearly dependent on IOP. The slope and the intercept estimated from the regression formula, for the eye phantom with $\mathrm{CCT}_{0}$ values of $0.37 \mathrm{~mm}, 0.46 \mathrm{~mm}$, and $0.57 \mathrm{~mm}$ are shown in Table 1 . It should be noted that the slope should be one if the current NCT readings are ideal for the estimation of the IOP inside eye phantoms. Both the slope and intercept of the plot increased with increasing $\mathrm{CCT}_{0}$. This may reflect the relative modulus changes accompanying changes in the $\mathrm{CCT}_{0}$. Thicker eye phantoms showed a higher NCT. The plot of NCT readings versus IOP, for fully continuous eye phantoms, with three different $\mathrm{CCT}_{0}$ are shown in Fig. 2(d). The slope and intercepts of the plot, shown in Table 1, increased with increasing $\mathrm{CCT}_{0}$, which was similar to that observed in fixed-cornea eye phantoms. It should be noted that, for all $\mathrm{CCT}_{0}$, the slope of the plot for fully continuous eye phantoms was less than that for fixed-cornea eye phantoms.

The stress and strain under IOP could be estimated from the data shown in Fig. 1(c)-(f). The stress-strain curves for cornea model eyes and full eyeball phantoms are shown in Fig. 3(a) and (b), respectively. In both model eyes, we could not recognize any apparent dependence of the slope on the $\mathrm{CCT}_{0}$. All the data were quite linear when the strain was small, which meant that the Young's modulus was independent of both IOP and $\mathrm{CCT}_{0}$.

From the stress-strain curves, slopes for the cornea model eye (Fig. 3(a)) and full eye phantom (Fig. 3(b)) were calculated $(594 \pm 16 \mathrm{kPa}$ and $623 \pm 12 \mathrm{kPa}$, respectively). Young's modulus of polydimethylsiloxane (PDMS) $(380 \mathrm{kPa})$, the material used to fabricate eye phantoms, was determined using a conventional tensile testing method. If both the slope of stress-strain curve and Young's modulus of PDMS were 
$>$ REPLACE THIS LINE WITH YOUR PAPER IDENTIFICATION NUMBER (DOUBLE-CLICK HERE TO EDIT) <

accurate, the range of Poisson's ratio were $0.36 \pm 0.02$ and $0.39 \pm 0.02$ for the cornea model eye and full eye phantom, respectively.

\section{DISCUSSION}

We investigated the structural deformation of the two different types of eye phantoms we developed with varying IOP. Further, NCT readings were also measured with an air-puff tonometer, which is the device mainly used in medical checkups for glaucoma screening, to obtain information on the changes in tension of the cornea from the outside. To analyze the structural changes of the eye phantoms quantitatively, we estimated the CCT and CRC from OCT images. We found that the dependence of the CCT and CRC on the IOP of the fully continuous eye phantoms differed from that of the fixed-cornea eye phantoms. Because parts other than the cornea were rigid in the fixed-cornea eye phantoms, only the corneas of the could be deformed by the changing IOP. Nevertheless, the CCT changes were very similar between the two models. In the case of the $\mathrm{CRC}$, the fixed-cornea eye phantom drastically decreased with increasing internal pressure (see Fig. 1 and Table 1). However, all parts of the fully continuous eye phantoms could be deformed. In these eye phantoms, we found the $\mathrm{CRC}$, as a function of changing IOP, was dependent on $\mathrm{CCT}_{0}$. When $\mathrm{CCT}_{0}$ was less than $0.46 \mathrm{~mm}$, the $\mathrm{CRC}$ decreased as IOP increased. However, when $\mathrm{CCT}_{0}$ was $0.57 \mathrm{~mm}$, the CRC increased with an increase in IOP.

When the IOP increases, deformation of the cornea should be mainly governed by its stiffness, which is determined by its material properties, structure, and boundary properties. At the boundary between the flexible and rigid parts of the fixed-cornea eye phantom, the cornea extended significantly as the applied pressure increased under the constraint condition. This caused the axial length of the eye phantom to increase and an eventual decrease in the CRC, accompanied by a decrease in CCT (Fig. 4(a)). In addition, the $\mathrm{CRC}$ was dependent on $\mathrm{CCT}_{0}$; more axially the cornea axially extended, the thinner it became. Among all fixed cornea eye phantoms, changes in the CRC of the fixed eyeball phantom with a $\mathrm{CCT}_{0}$ of $0.37 \mathrm{~mm}$ were most prominent. Thus, indicating that, in case of a thin cornea, IOP induces remarkable structural changes near the corneal apex. Meanwhile, the entire area of fully continuous eye phantoms could be deformed by IOP change even if the stiffness of the eyeball part, except the cornea, was relatively high. While the decrement of the CCT in the fully continuous eye phantom was comparable to that of the fixedcornea eye phantom, on applying pressure, the change in CRC in the fully continuous eye phantom was less prominent compared to the fixed-cornea eye phantom. When $\mathrm{CCT}_{0}$ was $0.57 \mathrm{~mm}$, the CRC was either constant or increased because most of the applied pressure contributed to compression of the CCT (Fig. 4(b)). Meanwhile, if the initial CCT was less than $0.46 \mathrm{~mm}$, the CRC of fully continuous eye phantoms decreased when the internal pressure increased (Fig. 4(c)).

It is of interest to discuss previous studies on the correlation between CCT, CRC, and IOP in the human eye. By collecting data from 1390 people, Shimmyo et al. reported a significant correlation between CCT and keratometric power, ${ }^{10}$ thicker eyes had flatter CRC, and thinner eyes had steeper CRC. In addition, by using enucleated human eyeballs, Hjortdal et al. ${ }^{13}$ reported an apparent increase in CRC with an increment in IOP. Although we have no information on the $\mathrm{CCT}_{0}$ of the subjects of the abovementioned studies, it led us to reasonably suppose that the fully continuous eye phantom with $\mathrm{CCT}_{0}$ of $0.57 \mathrm{~mm}$ is a more adequate model to describe the deformation of the human eye with an increase in IOP.

When the IOP increases, the corneal surface extends and the CCT decreases due to the force caused by the IOP. Assuming that we set the region of interest near the corneal apex, the system can be considered a part 
of thin-walled spherical shell. As the CCT is much less than the CRC, the circumferential stress can be expressed as follows ${ }^{14}$ :

$$
\sigma_{c}=\frac{I O P \times C R C}{2 \times C C T}
$$

Assuming that the volume of a finite element at the corneal apex is constant, when the strain is quite small, the circumferential strain, $\varepsilon_{c}$, can be expressed in terms of CCT as follows:

$$
\varepsilon_{C}=\left(\frac{C C T_{0}}{C C T}\right)^{1 / 2}-1
$$

Furthermore, Hooke's law states that

$$
\frac{E}{(1-v)}=\frac{\sigma_{c}}{\varepsilon_{c}}
$$

where E and $v$ are the Young's modulus and Poisson's ratio, respectively.

Even if the values of Poisson's ratio of the PDMS used in the construction of these eye phantoms remain rather limited, the values of full eyeball phantoms were close to the recently reported values in humans (range, $0.40-0.50)^{15}$. In case of the human cornea, the mean Poisson's ratio is $0.42^{16}$. Young's modulus (E) and shear modulus $(\mathrm{G})$ are related to $\mathrm{E}=2 \mathrm{G}(1+v)$ for isotropic and homogeneous materials. Therefore, the shear modulus can be estimated as approximately $93 \mathrm{kPa}$ and $91 \mathrm{kPa}$ for the cornea model eye and the full eye phantom, respectively. The values determined from our eye phantoms were close to the shear modulus of the human cornea of $\mathrm{G}=72 \pm 13.7 \mathrm{kPa}^{15}$. These observations led us to propose that our eye phantoms could be utilized to investigate deformation of the eye caused by IOP change or to measure the tension from the outside of the eyeball. Indeed, a recent study on biomechanics of an in vivo human cornea showed that the corneal shear modulus was independent of both CCT as well as IOP ${ }^{17}$.

We found that the tonometer readings of eye phantoms tends to increase with an increase in initial CCT. Even if an air-puff tonometer overestimates or underestimates the true IOP ${ }^{18}$, the observation from the current work is consistent with previous reports on the positive correlation between the measured tension from the outside of the eyeball and the $\mathrm{CCT}^{10}$. Meanwhile, the increment in the NCT readings, due to the elevation of the IOP from $5 \mathrm{mmHg}$ to $45 \mathrm{mmHg}$, in the fixed-cornea eye phantoms was approximately 1.5 times greater than that in fully continuous eye phantoms (Table 1). These discrepancies might be due to the difference in their structural deformation upon increasing the applied pressure. The CRC of the fixedcornea eye phantom decreased significantly as the IOP increased. This configuration makes the cornea harder to flatten, and eventually it exhibited higher NCT readings because the readings are derived from the force needed to flatten the cornea. In fully continuous eye phantoms, the changes in CRC with increasing IOP were less than those in fixed-cornea eye phantoms. Therefore, the ratio of NCT readings and IOP changes in fully continuous eye phantoms should be lower than that in fixed-cornea eye phantoms.

Finally, we evaluated which model eye best mimics the human eyeball, and is more suitable for understanding the relationship between the tension measured from outside and the structural deformation of the eyeball induced by an increment of IOP. It is not easy to measure both the CCT and CRC, which are correlated to structural deformation, in human subjects at an IOP of $0 \mathrm{mmHg}$. In addition, apart from enucleated human eyeball and large population data studies ${ }^{11,13}$, it is crucial to refer theoretical studies on deformation of the cornea upon applying IOP. A theoretical simulation study ${ }^{16}$ on the two types of eye models revealed an apparent increase in CRC with an increment in IOP in the fully continuous eye 
phantom with a relatively thick cornea. However, the CRC decreased, with an increment in IOP, in the fixed-cornea model. These considerations led us to safely conclude that fully continuous eye phantoms more closely recapitulated the structural deformation of the human eye by IOP change.

\section{CONClusion}

We conducted a comparative study on two different eye phantoms: fixed-cornea eye phantoms and fully continuous eye phantoms. First, we developed our own method to fabricate a fully continuous eye phantom. Subsequently, we measured the structural changes including CCT, CRC, and NCT readings with changes in IOP. In consideration of previous studies regarding structural deformation, from both in vitro experiments of the human eye and theoretical simulation, we found that our fully continuous eye phantom mimics the human eye more accurately. From the measured NCT data, we also found that NCT readings from fixed-cornea eye phantoms, in comparison to fully continuous eye phantoms, were overestimated. For these reasons, we expect that fully continuous eye phantoms could be suitable for the estimation of true IOP and allow for performance testing of tonometers, both for clinical use and medical checkup.

\section{METHODS}

\section{A. Fabrication of eye phantoms}

We developed two different types of eye phantoms. Fig. 5(a) and (b) show photographs of the fully continuous eye phantom and the fixed-cornea eye phantom, respectively. The detailed design of the phantoms, shown in Table 2, is based on the characteristics of the human eyeball, including its dimensional size $^{19,20}$, CCT ${ }^{10-12}$, diameter of the cornea ${ }^{12,21}$, and CRC ${ }^{10,12}$. The Young's modulus of the eye phantom was kept at approximately $380 \mathrm{kPa}$, which is close to that of the human cornea, by optimizing the composition of polydimethylsiloxane (PDMS; Sylgard 184, Dow Corning, the weight ratio of the silicone elastomer to the curing agent is $22: 1)^{22}$. The manufactured fully continuous eye phantom and the internals structure are shown in Fig. 5(c) and (d), respectively. The full eye phantom has a spherical shell structure and the shell is made up with PDMS and there is glycerol/water mixture in it as shown in Fig. 5(c).The cornea of the fixed-cornea eye phantom was fabricated by curing a mixture of base elastomer and curing agent inside an appropriate mold made of polyethylene terephthalate (PET); the fabricated cornea was fixed to the rigid components, including a water reservoir (Fig. 5(d)). Both initial CCT and CRC of the phantom were determined and the geometry of the mold was adjusted accordingly.

Meanwhile, CCT of the fully continuous eye phantom was controlled by varying the density of the glycerol/water mixture drop. The mold was made of PET. A liquid PDMS mixture was poured into the mold. Afterwards, the aqueous-glycerol solution was injected into the liquid PDMS mixture. Because the PDMS mixture is immiscible with the water-glycerol mixture, movement of the drop is governed by following three forces (see Fig. 5(e)).

Gravitational force: $F_{G}=\frac{\pi}{6} d^{3} \rho_{s} g$

Buoyancy force: $F_{B}=\frac{\pi}{6} d^{3} \rho g$

Drag force: $F_{D}=c_{d} \frac{1}{2} V^{2} \rho \mathrm{A}$

where $d$ is the diameter of the aqueous glycerol drop, $g$ is the gravitational acceleration, $\rho$ and $\rho_{s}$ is the density of the PDMS pre-polymer and aqueous glycerol solution, respectively. A is the projected area of the glycerol-water mixture drop, $c_{d}$ is the drag coefficient, and $\mathrm{V}$ is the velocity relative to the object. Under a steady state, the sum of the forces should be zero.

$F_{G}=F_{B}+F_{D}$ 
The drop of the glycerol-water mixture reaches the terminal velocity $\left(V_{t}\right)$, which can be expressed as follows:

$V_{t}=-\sqrt{\frac{4 g d}{3 C_{d}}\left(\frac{\rho_{s}-\rho}{\rho}\right)}$ when $\rho_{s}>\rho$

$V_{t}=\sqrt{\frac{4 g d}{3 C_{d}}\left(\frac{\rho-\rho_{s}}{\rho}\right)}$ when $\rho_{s}<\rho$

Both the magnitude and direction of movement of the drop can be controlled by varying the density of the glycerol-water mixture as well as its injected volume. The density of glycerol-water mixture was adjusted by changing its composition. The changes in the terminal velocity (Eq. (8) and (9)), according to the concentration of glycerol, can be represented as shown in Fig. 5(f). When the concentration of glycerol was $13 \%$, the terminal velocity of the drop was zero. The drop, whose density is greater than that of the PDMS mixture, tends to sink. If the drop is less dense than the liquid PDMS mixture, the buoyancy force can keep the drop afloat. Because the density as well as the drag coefficient of the liquid of PDMS pre-polymer depends on the degree of the cross-linking reaction, the terminal velocity of the drop also changes. Supposing that the curing condition is well designed and controlled for all the trials, to fabricate the phantom, vertically moving drop will be located at a specific point, of which the final position is only dependent on the nature of the drop. This means that the thickness between the bottom of the mold and the underside of the drop can be adjusted by varying both the density and volume of the drop of the glycerolwater mixture. Based on this principle, we made a fully continuous eye phantom, a three-dimensional model, in which the corneal thickness was adjustable.

The CCT of the eye phantoms was designed to be $0.35 \mathrm{~mm}, 0.45 \mathrm{~mm}$, and $0.55 \mathrm{~mm}$; these values were close to the average CCT of the human eyeball. After fabrication, we found that CCT, when no force was applied, was $0.37 \mathrm{~mm}, 0.46 \mathrm{~mm}$, and $0.57 \mathrm{~mm}$. In addition, considering the human CRC, the CRC of 8.5 $\mathrm{mm}$ was designed. Young's modulus of the PDMS phantom materials was adjusted to approximately 380 $\mathrm{kPa}$ by controlling the composition of the liquid PDMS mixture. The specifications of these two types of eye phantoms are compiled in Table 2.

\section{B. Experimental setup for controlling IOP}

A syringe pump-based pressure control system (C\&V Tech, Korea) was used to control the internal pressure of the eye phantom. The fixed-cornea eye phantom was connected to pressure control system through a tube (Fig. 5(g)). The IOP for fully continuous eye phantoms were adjusted by directly injecting the water using a needle (Fig. 5(h)). The height at top of the eye phantom was kept same as that of the syringe in order to maintain the same pressure. IOP inside the eye phantom was measured using a calibrated pressure sensor (PSH-B0200, Sensys), the measurement accuracy of which was less than $0.15 \%$.

\section{Measurement}

To examine the corneal deformation, tomographic images were obtained with a spectral domain optical coherence tomography (SD-OCT) system (Telesto, Thorlabs, USA) as shown in Fig. 5(i). The center wavelength and wavelength range were $1300 \mathrm{~nm}$ and $100 \mathrm{~nm}$, respectively. We scanned the cornea with Galvano scanners at a frame rate of approximately $18 \mathrm{f} / \mathrm{s}$. The range of B-scan, which was made up of 4096 A-scans, was $10 \mathrm{~mm}$. The A-scan had 512 pixels, depth of $2.528 \mathrm{~mm}$, and a refractive index of 1.00 . The depth resolution was approximately $4.9 \mu \mathrm{m}$. Both CCT and CRC were obtained by analyzing the OCT images with a data processing code written in Python.

The anterior and posterior surfaces of the cornea, from the OCT image, were extracted by using the edge detection algorithm. The CCT was estimated by dividing the distance between the two surfaces at the apex of the cornea by 1.4, the refractive index of PDMS (Fig. S1(a)). CRC was estimated by fitting the anterior surfaces with a circular function (Fig. S1(b)). An air-puff tonometer (CT-80, Topcon) was used to collect NCT readings of the eye phantom by varying the IOP from $0 \mathrm{mmHg}$ to $45 \mathrm{mmHg}$ as shown in Fig. 5(j). 
> REPLACE THIS LINE WITH YOUR PAPER IDENTIFICATION NUMBER (DOUBLE-CLICK HERE TO EDIT) <

The direction of the air jet was kept perpendicular to the corneal surface. NCT readings were averaged from three different measurements.

\section{Data availability}

All data generated or analysed during this study are included in this published article and its Supplementary Information files.

\section{Acknowledgements}

This work was supported in part by the Korea Research Institute of Standards and Science (grant 20011274) and the Ministry of Science and ICT(MSIT) (No. 2018M3A9H6081482).

\section{Author Contributions}

H.S.C. and S.C.J. designed the study, developed the phantoms, collect and analyze the data. H.S.C and S.C.J also wrote the manuscript with the collected data.

\section{Competing Interests}

The author(s) declare no competing interests.

\section{REFERENCES}

1. Gallab, M. et al. Development of a spherical model with a 3D microchannel: An application to glaucoma surgery. Micromachines 10, 1-12 (2019).

2. Fogli, G. et al. New eye phantom for ophthalmic surgery. J. Biomed. Opt. 19, 068001 (2014).

3. Arianpour, A. et al. An optomechanical model eye for ophthalmological refractive studies. $J$. Refract. Surg. 29, 126-132 (2013).

4. Rowe, T. S. \& Zawadzki, R. J. Development of a corneal tissue phantom for anterior chamber optical coherence tomography (AC-OCT). Des. Perform. Valid. Phantoms Used Conjunction with Opt. Meas. Tissue V 8583, 85830I (2013).

5. Kim, H. J., Seo, Y. H. \& Kim, B. H. New intraocular pressure measurement method using reflected pneumatic pressure from cornea deformed by air puff of ring-type nozzle. PLoS One 12, 1-15 (2017).

6. Deluthault, A. et al. Efficient Objective Metric Tool for Medical Electrical Device Development: Eye Phantom for Glaucoma Diagnosis Device. J. Sensors 2017, 8436123 (2017).

7. Hsu, S. et al. An RFID-based on-lens sensor system for long-term IOP monitoring. in 2015 37th Annual International Conference of the IEEE Engineering in Medicine and Biology Society (EMBC) 7526-7529 (2015). doi:10.1109/EMBC.2015.7320133.

8. Khan, A. A., Cortina, S. M., Chamon, W. \& Royston, T. J. Elastography methods applicable to the eye. Opt. Elastography Tissue Biomech. 8946, 89460B (2014).

9. Ko, M. W. L., Leung, L. K. K., Lam, D. C. C. \& Leung, C. K. S. Characterization of corneal tangent modulus in vivo. Acta Ophthalmol. 91, 263-269 (2013).

10. Shimmyo, M. Intraocular pressure, goldmann applanation tension, corneal thickness, and corneal curvature in caucasians, asians, hispanics, and african americans. Am. J. Ophthalmol. 137, 1170 (2004).

11. Dimasi, D. P. et al. Ethnic and mouse strain differences in central corneal thickness and association with pigmentation phenotype. PLoS One 6, (2011).

12. Mashige, K. P. A review of corneal diameter, curvature and thickness values and influencing factors*. African Vis. Eye Heal. 72, 185-194 (2013). 
$>$ REPLACE THIS LINE WITH YOUR PAPER IDENTIFICATION NUMBER (DOUBLE-CLICK HERE TO EDIT) <

13. Hjortdal, J. O. \& Jensen, P. K. In vitro measurement of corneal strain, thickness, and curvature using digital image processing. Acta Ophthalmologica Scandinavica vol. 73 5-11 (1995).

14. Hibbeler, R. C. Mechanics of Materials. (Pearson Prentice Hall, 2011).

15. Dogru, S., Aksoy, B., Bayraktar, H. \& Alaca, B. E. Poisson's ratio of PDMS thin films. Polym. Test. 69, 375-384 (2018).

16. Uchio, E., Ohno, S., Kudoh, J., Aoki, K. \& Kisielewicz, L. T. Simulation model of an eyeball based on finite element analysis on a supercomputer. Br. J. Ophthalmol. 83, 1106-1111 (1999).

17. Ramier, A. et al. In vivo measurement of shear modulus of the human cornea using optical coherence elastography. Sci. Rep. 10, 1-10 (2020).

18. Harper, R. \& Henson, D. B. Chapter 8 - Diagnosis of the glaucomas 2: intraocular pressure. in Glaucoma Identification \& Co-management (eds. Edgar, D. F. \& Rudnicka, A. R.) 107-118 (Butterworth-Heinemann, 2007). doi:https://doi.org/10.1016/B978-0-7506-3782-4.50011-9.

19. Bekerman, I., Gottlieb, P. \& Vaiman, M. Variations in Eyeball Diameters of the Healthy Adults. $J$. Ophthalmol. 2014, 1-5 (2014).

20. Snell, R. S. \& Lemp, M. A. The Eyeball. in Clinical Anatomy of the Eye 132-213 (John Wiley \& Sons, Ltd, 2013). doi:10.1002/9781118690987.ch6.

21. Baumeister, M., Terzi, E., Ekici, Y. \& Kohnen, T. Comparison of manual and automated methods to determine horizontal corneal diameter. J. Cataract Refract. Surg. 30, 374-380 (2004).

22. Hamilton, K. E. \& Pye, D. C. Young's modulus in normal corneas and the effect on applanation tonometry. Optom. Vis. Sci. 85, 445-450 (2008).

\section{Figure legends}

Figure 1. The OCT images of the fixed-cornea eye phantom (a) and the continuous eye phantom (b) observed with change in applied pressure. The $\mathrm{CCT}_{0}$ of the two phantoms is $0.37 \mathrm{~mm}$. The scale bar is 0.5 $\mathrm{mm}$. Arrows of the same color is the same size. The dependence of the CCT on the applied pressure for the fixed-cornea eye phantom (c) and the fully continuous eye phantom (d). The changes in CRC for the fixedcornea eye phantom and the fully continuous one are shown in (e) and (f), respectively.

Figure 2. The experimental setup for measuring IOP with a non-contact tonometer for the fixed-cornea eye phantom (a) and the fully continuous eye phantom (b). The relationship between the NCT reading and the IOP for each eye phantom are shown in (c) and (d).

Figure 3. Stress-strain curves for cornea model (a) and full eyeball phantoms (b) derived from the data shown in Fig. 1(c)-(f). The black solid lines are the results of the linear regression conducted on all the data

Figure 4. Illustration describing the structural deformation caused by the IOP increment for the fixedcornea eye phantom (a) and the fully continuous eye phantom with thick (b) and thin (c) $\mathrm{CCT}_{0}$.

Figure 5. The photographs of fully continuous eye phantom (a) and fixed-cornea eye phantom (b). The crosssectional views of the two eye phantoms are shown in (c) and (d). The diagram of a glycerol-water mixture drop in PDMS pre-polymer (e). In this case, the density of glycerol-water mixture $\left(\rho_{s}\right)$ is higher than the density of PDMS pre-polymer $(\rho)$. The relationship between glycerol concentration of glycerol-water mixture and the velocity of the glycerol-water mixture drop in PDMS pre-polymer when the ambient temperature is $20{ }^{\circ} \mathrm{C}$ and $25{ }^{\circ} \mathrm{C}$ (f). When the glycerol concentration is near $13 \%$, there is a singular point to change the moving direction of the glycerol-water mixture drop. The syringe pump-based pressure control system for the fixed-cornea eye phantom ( $\mathrm{g}$ ) and fully continuous eye phantom (h). The schematic diagram for measuring the structural deformation and NCT readings of the cornea are shown in (i) and (j), respectively. 
Tables

Table 1. A comparison of the total changes of CCT and CRC among the initial targeted CCT and the types of the eye phantoms and a comparison of the difference of the NCT with the different initial CCT for the two different types of eye phantoms

\begin{tabular}{|l|c|c|c|c|}
\hline \multirow{2}{*}{$\begin{array}{c}\mathbf{C C T}_{\mathbf{0}} \\
(\mathbf{m m})\end{array}$} & \multicolumn{2}{|c|}{$\begin{array}{c}\text { Slope of the plot of CCT to IOP } \\
(\boldsymbol{\mu m} / \mathbf{m m H})\end{array}$} & \multicolumn{2}{c|}{ Total change of CRC (mm) } \\
\cline { 2 - 5 } & $\begin{array}{c}\text { Fixed-cornea eye } \\
\text { phantom }\end{array}$ & $\begin{array}{c}\text { Fully continuous } \\
\text { eye phantom }\end{array}$ & $\begin{array}{c}\text { Fixed-cornea eye } \\
\text { phantom }\end{array}$ & $\begin{array}{c}\text { Fully continuous } \\
\text { eye phantom }\end{array}$ \\
\hline $\mathbf{0 . 3 7}$ & -1.87 & -1.84 & -3.21 & -0.93 \\
\hline $\mathbf{0 . 4 6}$ & -1.59 & -1.79 & -2.79 & -0.51 \\
\hline $\mathbf{0 . 5 7}$ & -1.7 & -1.96 & -2.42 & 0.17 \\
\hline $\mathbf{C C T}_{\mathbf{0}}$ & Fixed-cornea eye phantom & \multicolumn{2}{c|}{ Fully continuous eye phantom } \\
\cline { 2 - 5 }$(\mathbf{m m})$ & Slope & Intercept $(\mathbf{m m H g})$ & Slope & Intercept (mmHg) \\
\hline $\mathbf{0 . 3 7}$ & 0.744 & -0.598 & 0.552 & 2.04 \\
\hline $\mathbf{0 . 4 6}$ & 0.887 & 4.06 & 0.577 & 5.98 \\
\hline $\mathbf{0 . 5 7}$ & 0.950 & 10.4 & 0.611 & 12.1 \\
\hline
\end{tabular}

Table 2. The features of the two types of eye phantoms

\begin{tabular}{|l|c|c|}
\hline \multicolumn{1}{|c|}{ Feature } & Fully continuous eye phantom & Fixed-cornea eye phantom \\
\hline Central corneal thickness $(\mathbf{m m})$ & $0.37-0.57$ & $0.37-0.57$ \\
\hline Young's modulus (kPa) & 380 & 380 (only cornea) \\
\hline $\begin{array}{l}\text { Corneal radius of curvature } \\
(\mathbf{m m})\end{array}$ & 8.5 & $8-9$ \\
\hline Materials of eye phantoms & PDMS & $\begin{array}{c}\text { Cornea: PDMS } \\
\text { Other parts: rigid plastic }\end{array}$ \\
\hline
\end{tabular}




\section{Figures}
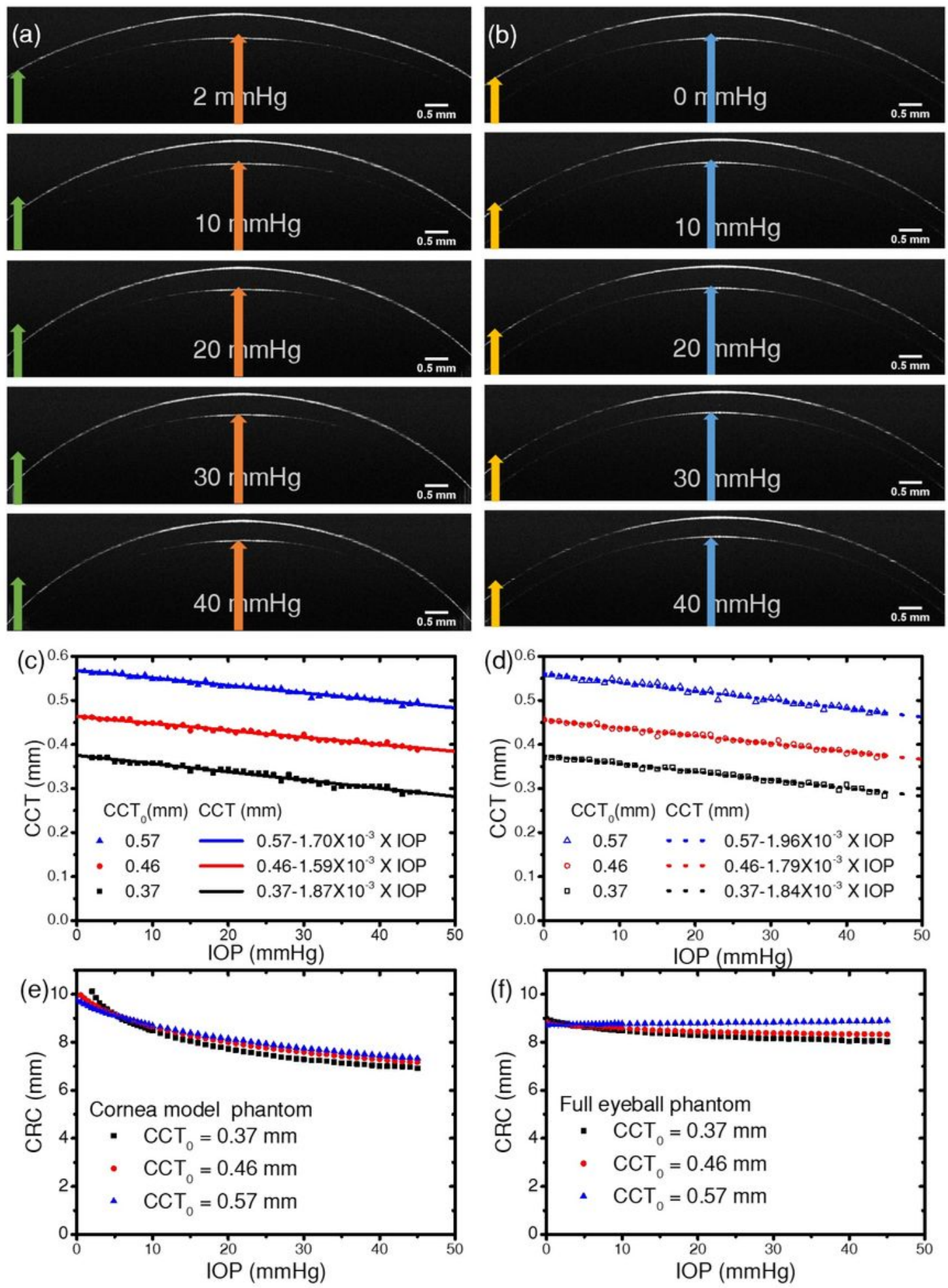

\section{Figure 1}

The OCT images of the fixed-cornea eye phantom (a) and the continuous eye phantom (b) observed with change in applied pressure. The СCT0 of the two phantoms is $0.37 \mathrm{~mm}$. The scale bar is $0.5 \mathrm{~mm}$. Arrows of the same color is the same size. The dependence of the CCT on the applied pressure for the fixed- 
cornea eye phantom (c) and the fully continuous eye phantom (d). The changes in CRC for the fixedcornea eye phantom and the fully continuous one are shown in (e) and (f), respectively.
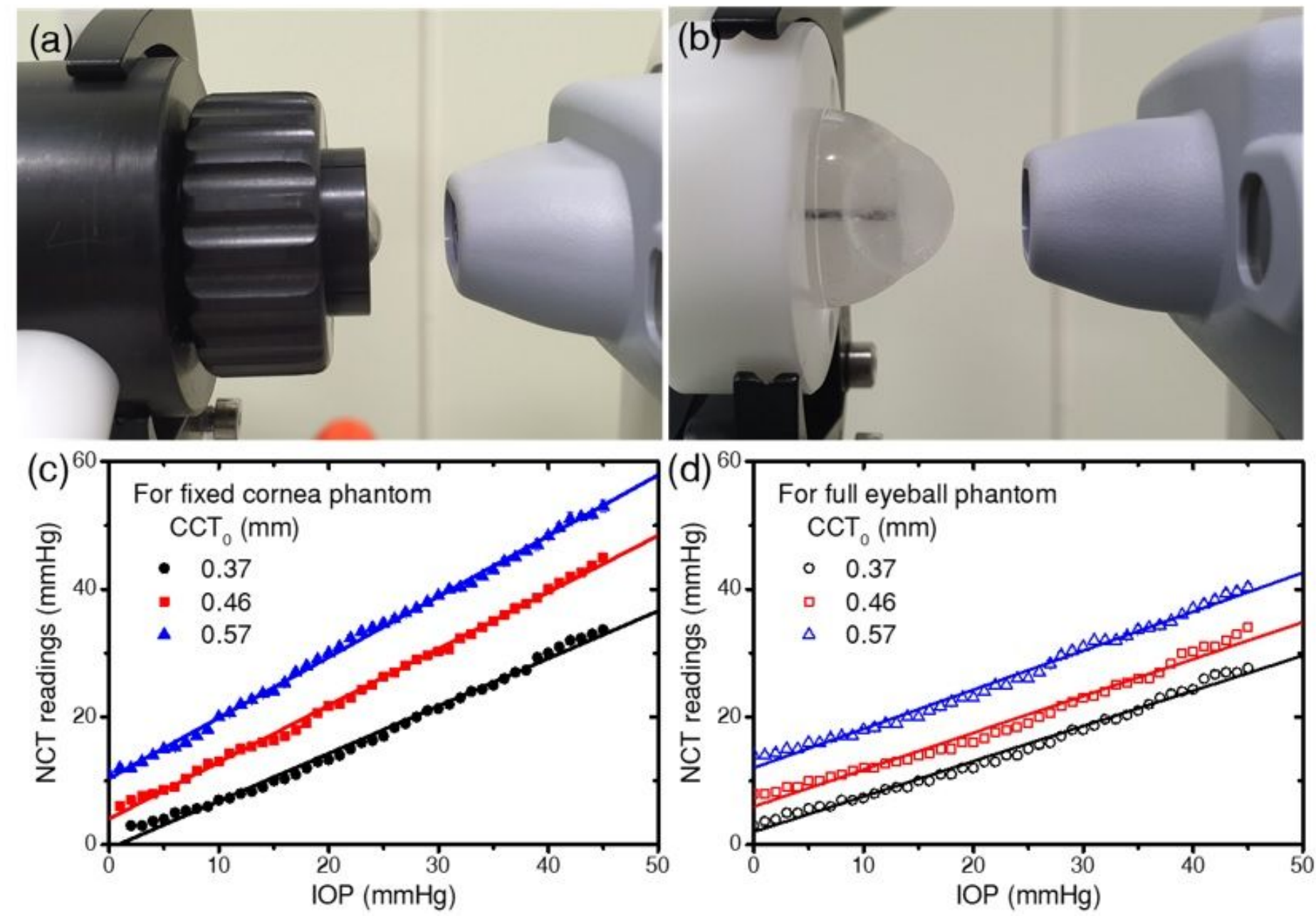

\section{Figure 2}

The experimental setup for measuring IOP with a non-contact tonometer for the fixed-cornea eye phantom (a) and the fully continuous eye phantom (b). The relationship between the NCT reading and the IOP for each eye phantom are shown in (c) and (d).
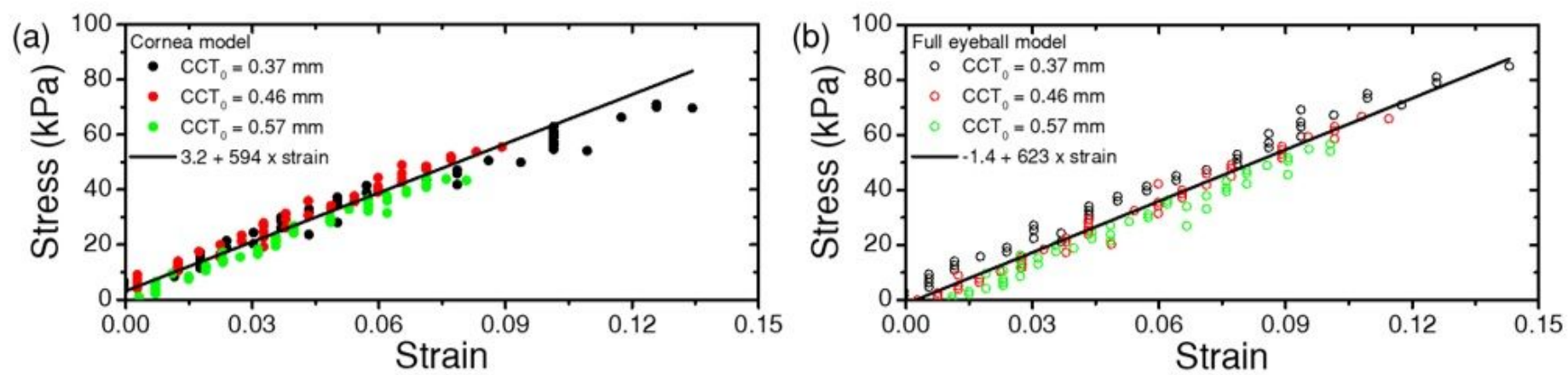

Figure 3 
Stress-strain curves for cornea model (a) and full eyeball phantoms (b) derived from the data shown in Fig. 1(c)-(f). The black solid lines are the results of the linear regression conducted on all the data

(a)
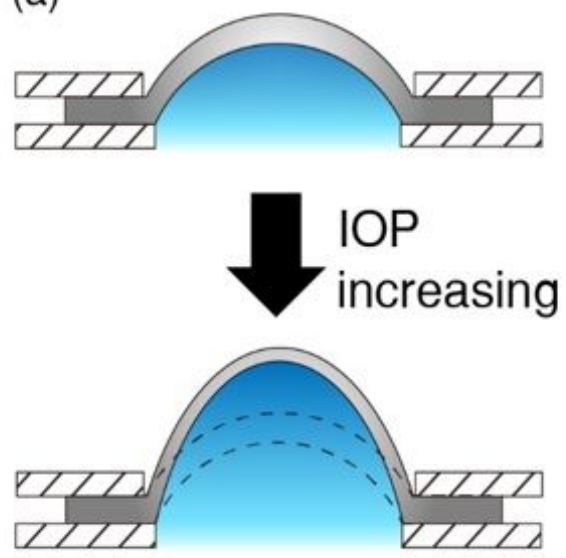

Solid part

(b)
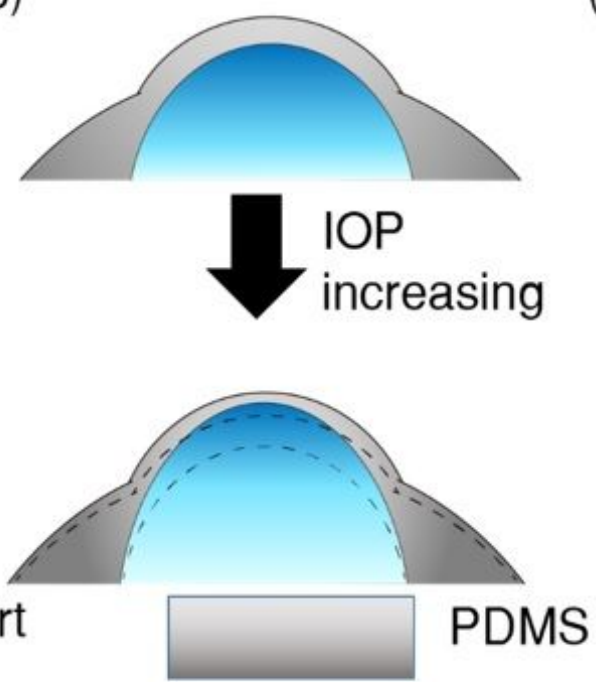

(c)

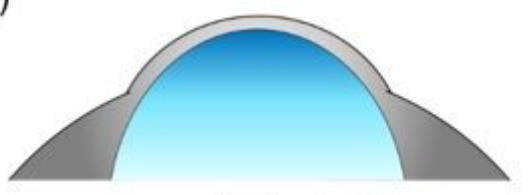

$\checkmark \begin{aligned} & \text { IOP } \\ & \text { increasing }\end{aligned}$

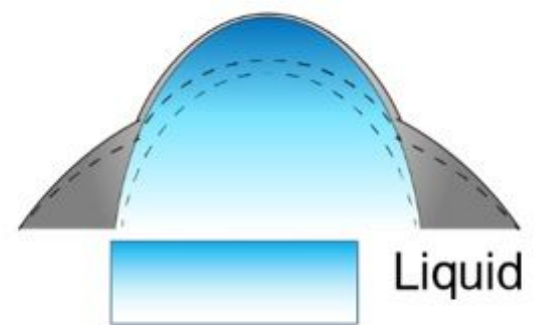

\section{Figure 4}

Illustration describing the structural deformation caused by the IOP increment for the fixed-cornea eye phantom (a) and the fully continuous eye phantom with thick (b) and thin (c) ССТ0. 


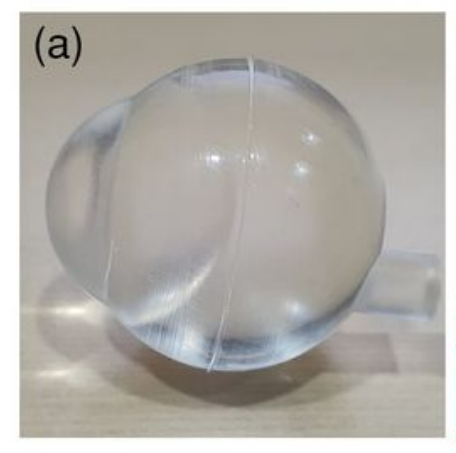

(e)
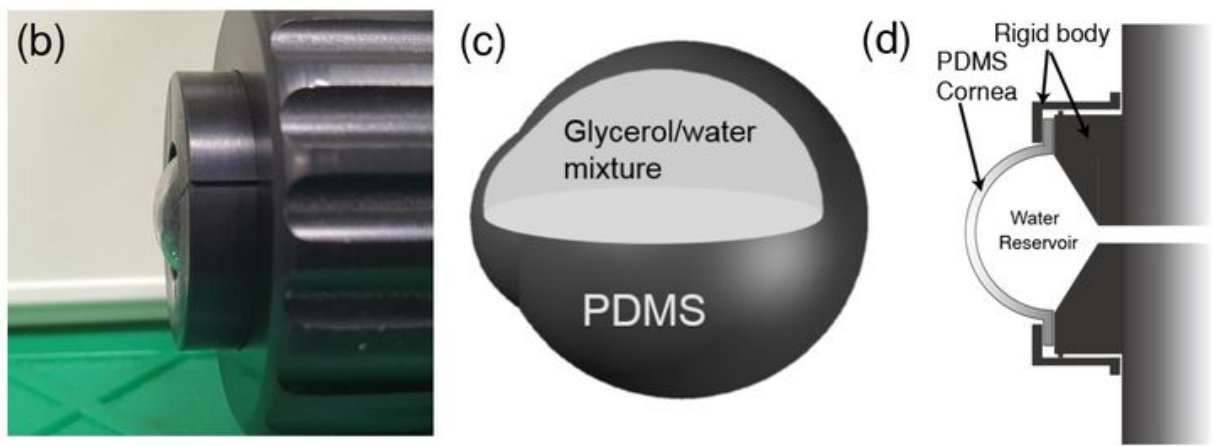

(f)

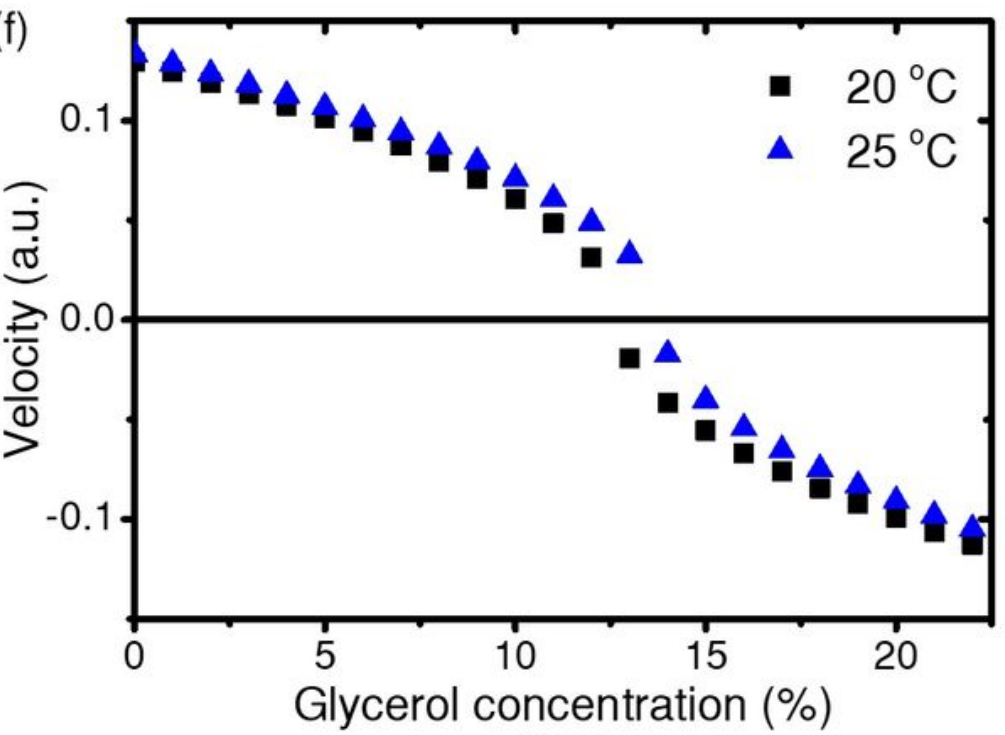

(g)

PDMS

pre-polymer $(\rho)$

$F_{D}+F_{B}$

Glycerol

-water

mixture $\left(\rho_{\mathrm{s}}\right)$

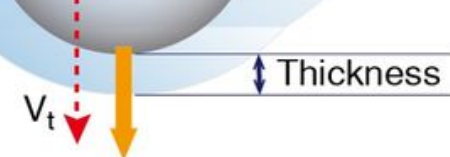

$F_{G}$

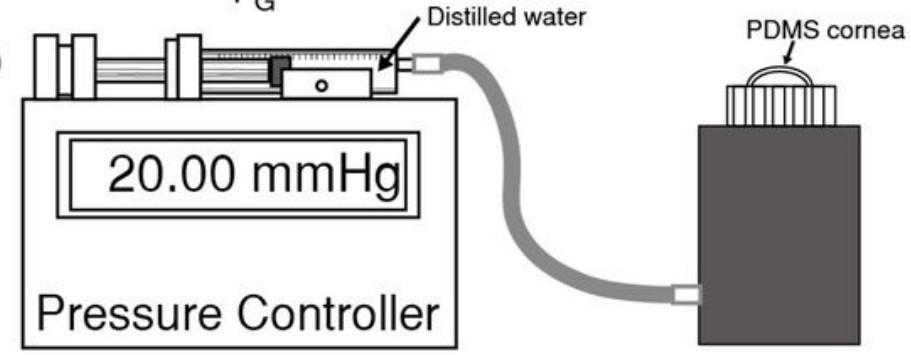

(h)

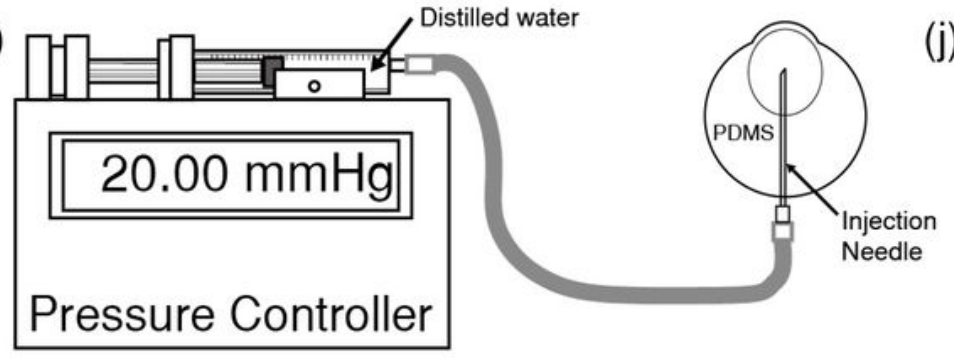

(i)

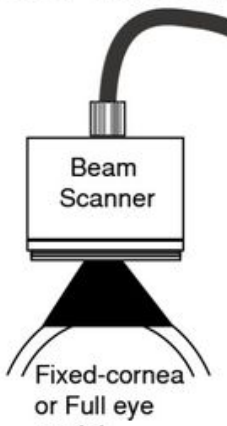

(j)

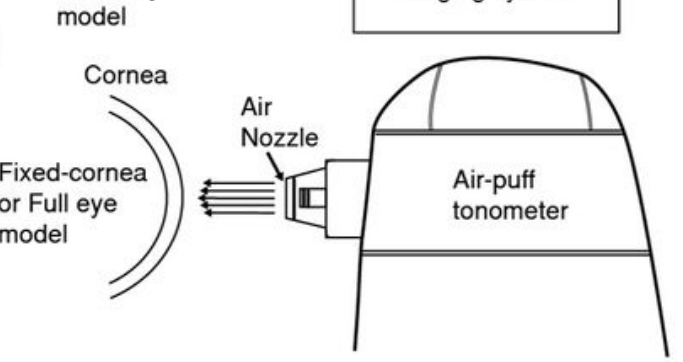

\section{Figure 5}

The photographs of fully continuous eye phantom (a) and fixed-cornea eye phantom (b). The crosssectional views of the two eye phantoms are shown in (c) and (d). The diagram of a glycerol-water mixture drop in PDMS pre-polymer (e). In this case, the density of glycerol-water mixture ( $\rho s)$ is higher than the density of PDMS pre-polymer $(\rho)$. The relationship between glycerol concentration of glycerolwater mixture and the velocity of the glycerol-water mixture drop in PDMS pre-polymer when the ambient 
temperature is $20^{\circ} \mathrm{C}$ and $25^{\circ} \mathrm{C}$ (f). When the glycerol concentration is near $13 \%$, there is a singular point to change the moving direction of the glycerol-water mixture drop. The syringe pump-based pressure control system for the fixed-cornea eye phantom (g) and fully continuous eye phantom (h). The schematic diagram for measuring the structural deformation and NCT readings of the cornea are shown in (i) and (j), respectively.

\section{Supplementary Files}

This is a list of supplementary files associated with this preprint. Click to download.

- SciRepSupplHSChoSCJEOUNG20210210clean.docx 